Please send trade news information and

illustrations to Arveen Bajaj at the $B D J$, Nature

Publishing Group, The Macmillan Building,

4-6 Crinan Street, London N1 9XW.

Trade news is provided as a service to readers

using text and images from the manufacturer,

supplier or distributor and does not imply

endorsement by the BDJ. Normal and prudent

research should be exercised before purchase or

use of any product mentioned.

\section{Keeping children entertained}

The new Admor Interiors Directory showcases a range of reception furniture and activity products to prevent your younger patients from getting bored in the waiting room and to keep them looking forward to their next visit to the dentist.

The Animal furniture group is very popular with younger children and features robust, fun and colourful rubber wood furniture available with frog, teddy bear, mouse or cat designs on the backrests.

If floor space is limited in your patient waiting room, Admor can supply a space-saving, wall-mounted activity panel that is the ideal way to keep two or three children occupied at one time. The Wires, Beads and Gears Panel has no removable parts, ensuring it can be kept in good condition and that nothing goes missing.

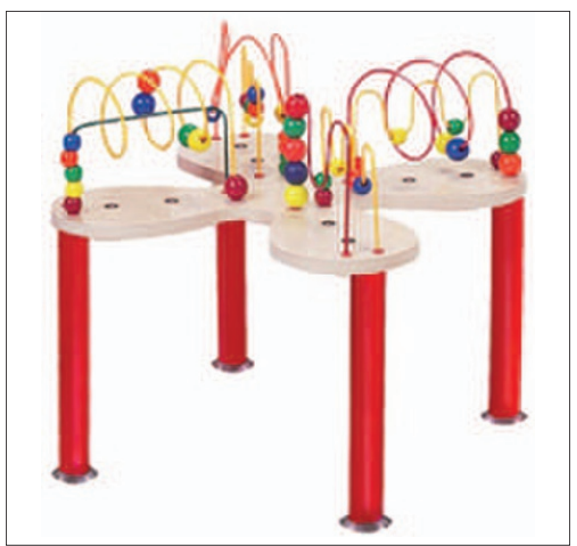

The new Mini Curves and Waves Table is a brightly coloured wooden table supporting a range of curvy bead tracks. Children will love gathering around the smooth-edged table and racing the chunky wooden beads.

It is hoped that the products which are available in Admor's 2007 catalogue will act as an incentive for children to visit the surgery.

Reader response number 50

\title{
Safe operation on bone tissue
}

Surgison is the new ultrasound handpiece from Castellini, which allows safe and effective operation on bone tissue with a high-power, high-performance instrument. Cutting is much cleaner and more precise compared to a rotary instrument and the rise in bone tissue temperature is significantly limited.

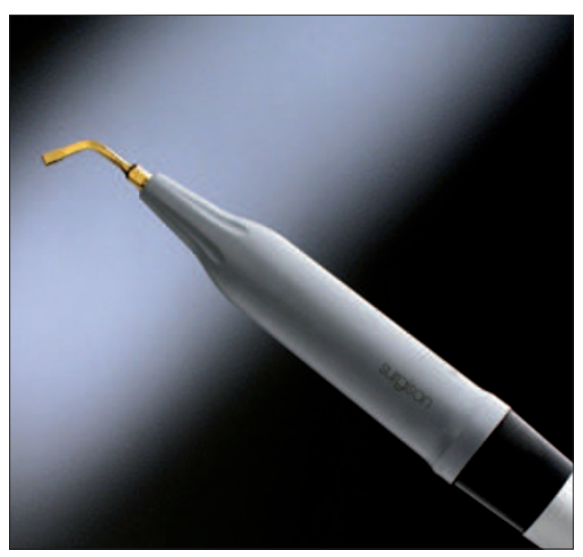

The cutting action of the Surgison is effective only on bone tissues; other frequencies would be required for the cutting of soft tissue and this is an especially beneficial quality that makes way for safe operation on bone tissues in close proximity to delicate vascular and nerve structures. It can be used for oral, implant, periodontal, orthodontic, enzootic and extractive surgery.

Surgison is available with Castellini Logos and Duo dental units and has been designed especially for compatibility with the previous models of these dental units as well as the updated models. Once installed onto the dental unit, the Surgison is simply connected to the same hose as the normal scaler - the dental unit's electronics then recognise the surgical instrument connected and select the correct control system.

Reader response number 51

\section{New docking station}

Sirona Dental Systems has introduced a new docking station in order to further enhance the userfriendliness of the SiroLaser system. This docking station ensures that the diode laser and the handpiece are always at the user's fingertips.

Various mounting options are available and in addition, the fibre optic cable is now supplied together with a compatible spool system. This allows the dentist to fix the cable in any chosen position and avoid possible damage to the sensitive fibres.

Due to its modular design, the docking station can be adapted to any treatment centre model. With the help of the single and double Sirodoc mounting brackets, the handpiece and the spool can be attached either laterally or centrally. If required, the dentist can also mount the spool at the rear of the Sirodoc unit.

Irrespective of its position, there are various options for connecting the spool to the Sirodoc unit (on its side, upright or in a hanging position). The SiroLaser is very compact $(87 \mathrm{~mm} \times 54 \mathrm{~mm} \times 190$ $\mathrm{mm}$ ) and weighs $450 \mathrm{~g}$. As a result it is easily transportable from one treatment room to another.

Reader response number 52

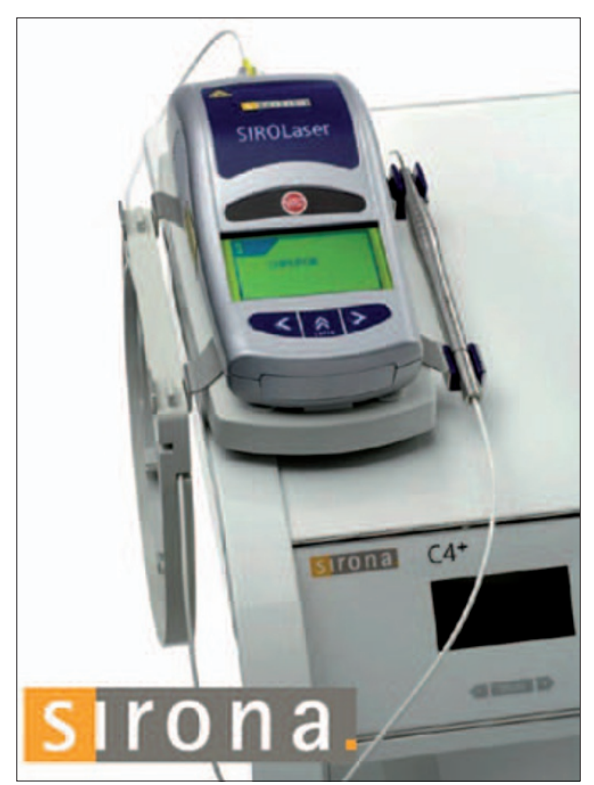




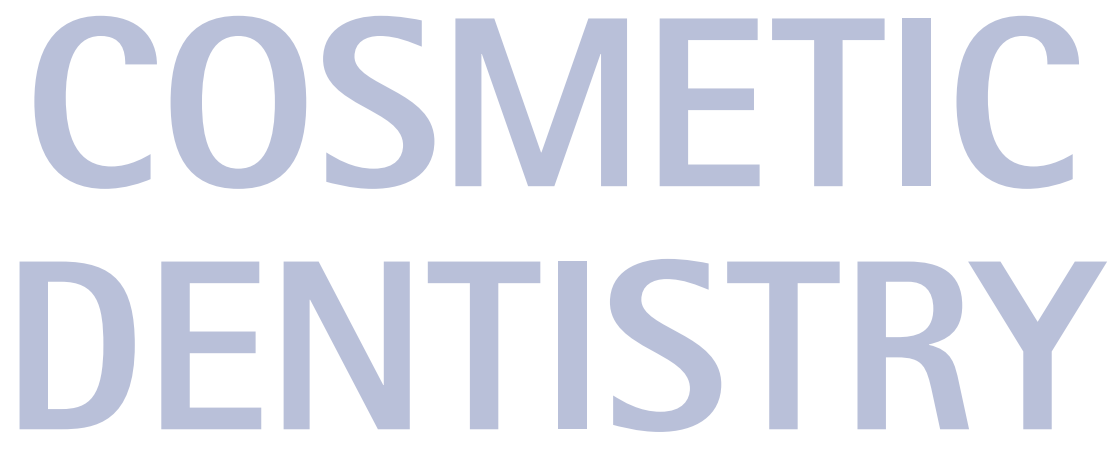

\section{A solution to aesthetic orthodontics}

SPA Brackets from DB Orthodontics are clear brackets manufactured from zirconium mono-sapphire which will not discolour, allowing the natural aesthetics of individual teeth to show through.

A special heat treatment results in strength and break resistance. At only $2 \mathrm{~mm}$ high, they have a low profile and are only slightly larger than conventional stainless steel brackets. They also feature rounded margins and corners for even greater comfort.

Supplied in either Roth .022 or MBT prescriptions, they have an optimised slot design with a two point corner contact. The slot is highly polished and the reduced frictional resistance results in good sliding mechanics.

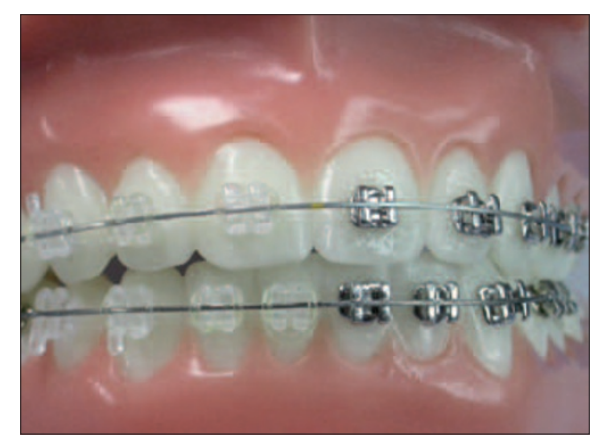

The base design features numerous micro-protuberances and has a special surface treatment which results in superior bond strength, yet protects the enamel from damage during de-bonding. Compatible with all the most popular brands of orthodontic adhesives, SPA Brackets are easy to de-bond, just like metal brackets.

Reader response number 53

\section{Effective whitening system}

Illuminé from Dentsply is a simple and effective whitening system that you can incorporate into your practice almost immediately, as no light or heat equipment is required.

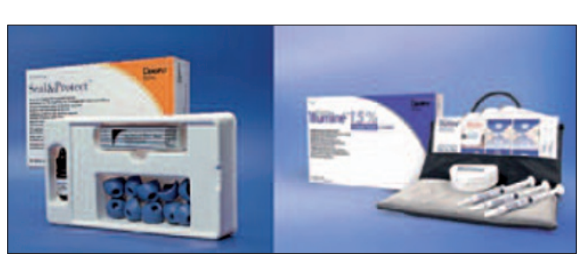

In addition, Dentsply also offers SealetProtect, a protective sealant for exposed dentine. It is simply brushed on to the area that has been exposed due to abrasion or erosion, and then forms a protective barrier to stop the pain.

The protective effect of SealctProtect usually lasts around 12 months, resists acids in food and beverages and helps towards reducing sensitivity when whitening teeth.

Reader response number 54

\section{Treating tooth sensitivity}

Blackwell Supplies now offers Denshield to treat tooth sensitivity. Denshield offers patients relief from sensitivity both before and after bleaching and is designed to reduce sensitivity and treat the cause of the problem, providing rapid and continuous relief and actively reducing open dentine tubules to prevent the exposure of the pulp nerve to stimuli.

Denshield can be applied directly to sensitive areas to completely seal any open tubules and provide immediate short-term relief. Patients can then continue the treatment at home to enjoy the benefits of long-term active protection of the tooth nerve.

Reader response number 55

\section{All-ceramic restorations}

Ivoclar Vivadent offers a choice of allceramic options in four new bleach shades, enabling dentists to provide their patients with all-ceramic restorations for every indication.

The IPS e.max system enables dentists to provide single or multiple unit all-ceramic restorations using a range of high strength materials including zirconium oxide, the final restorations offering a balance between strength, colour saturation and translucency. Dentists can provide their patients with the benefits of IPS e.max either at the chairside using IPS e.max CAD LT blocks or indirectly via their laboratory.

The IPS Empress system is another popular choice for crowns, inlays, onlays and veneers. It is available as IPS Empress Esthetic, the laboratory pressed ceramic system and IPS Empress CAD blocks for use with Cerec and InLab CAD/CAM technology. Both IPS e.max and IPS Empress are available in a wide number of A-D shades including four new BL Bleach shades.

Reader response number 56

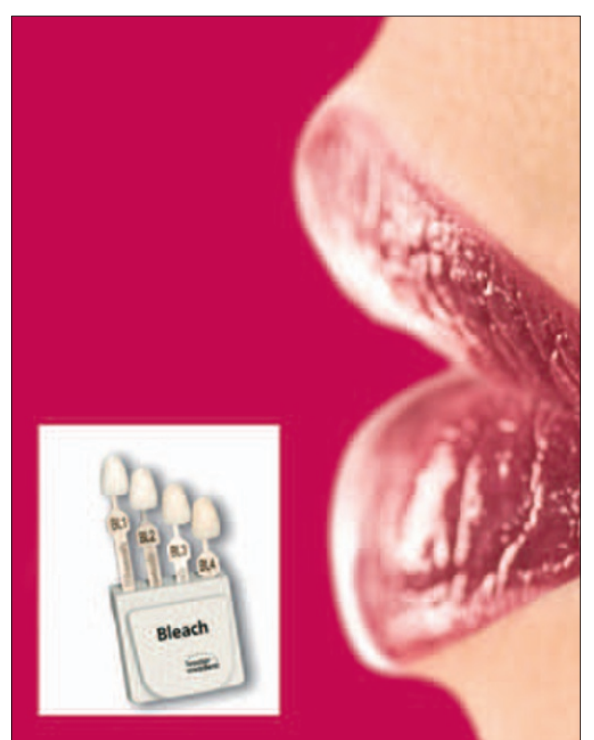




\section{An alternative to amalgam}

Trycare Dental Linkline's Alert condensable composite is an alternative to amalgam which enables dentists to provide high-grade posterior restorations quickly and easily.

Alert is a highly filled poly-ceramic material containing conventional fillers plus chopped glass fibres. Packing like amalgam, during condensing it exhibits no tackiness, pull back or slumping and allows the operator to recreate original form and contact points quickly and easily.

With a low 1.2\% shrinkage rate and over $5 \mathrm{~mm}$ depth of cure, Alert can be used safely to bulk fill restorations.

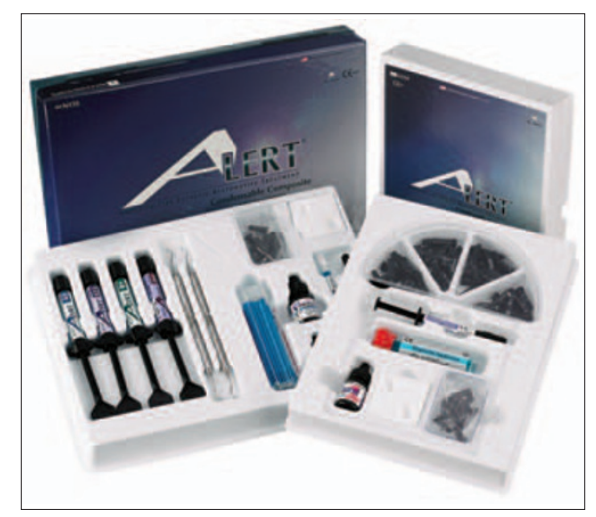

Allied to its high flexural strength and knoop hardness, Alert is a hard wearing yet tooth friendly restorative and is supplied in five Vita matching shades (A2, A3, A3.5, B1, and C2 ).

Reader response number 57

\section{Range of tooth}

\section{whitening products}

Dental Sky supplies a complete range of tooth whitening products from leading manufacturers from its latest catalogue. The company is a supplier of brands such as Illumine from Dentsply or Pola from SDI. Within Dental Sky's portfolio is the new Velopex Diode Laser, which is recommended for laser activated whit- ening procedures. The Vita EasyShade from Panadent allows you to accurately and simply compare the shade of your patients' teeth before and after tooth whitening.

Also offered is the Dentoid Video Headset. This piece of equipment allows your patient to watch their favourite movie, whilst having their teeth whitening in the surgery, whether they are sitting upright or lying down.

Reader response number 58

\section{Match the colour concept}

GC's Gradia Direct Flo flowable composite is ideal for use in conjunction with Gradia Direct and the shades match the colour concept of the easy to use and aesthetic composite.

Due to its physical properties and flow characteristics Gradia Direct Flo can be used as a liner, for blocking undercuts under composite restorations and for minimal intervention cavities.

Its non-running and non-stick formula shapes to cavity walls and makes it easy to handle. The radiopaque material is supplied in a syringe design that

\section{New dental implant}

Biomet 3i, manufacturers of oral reconstructive products including dental implant components and bone and tissue regenerative materials have launched the new NanoTite Implant which has Osseotite Surface at the core.

Discrete nanometer scale crystals of calcium phosphate (CaP) are deposited onto the Osseotite Surface substrate and are bonded to it, occupying approxi-

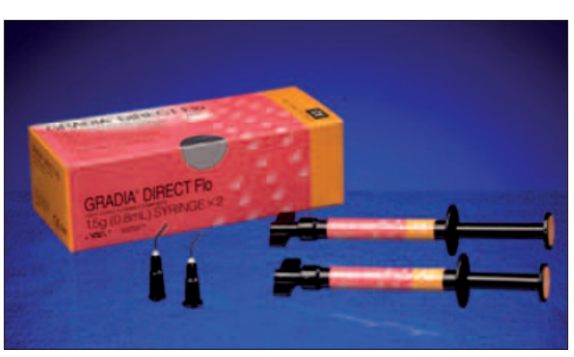

allows for easy manipulation and precise application. It is available in seven shades, A1, A2, A3, A3.5, A03, CV and BW.

Reader response number 59

mately 50\% of the surface. The implant is available in two configurations. The NanoTite Certain Implant is a modified hybrid where the coverage of both the Osseotite Surface and the nano-scale crystals go up to the base of the implant collar. The NanoTite Prevail Implant incorporates integrated Platform Switching and has the Osseotite Surface and the nano-scale crystals to the top of the collar, creating a continuous bone-loading surface.

Reader response number 60 